\title{
EDITORIAL
}

\section{The changing face of meningococcal disease in West Africa}

\section{B. GREENWOOD*}

Department of Infectious and Tropical Diseases, London School of Hygiene \& Tropical Medicine, London, UK

(Accepted 2 May 2007; first published online 20 June 2007)

Just over 100 years ago the first epidemic of meningococcal meningitis was described in Africa [1]. This epidemic probably started in northern Nigeria and spread rapidly westwards reaching present-day Ghana in 1906 where its epidemiological features including its size, high attack rate and seasonality were well described by Horn [2]. During the subsequent 100 years large epidemics of meningococcal meningitis have occurred every few years in countries of the Sahel and sub-Sahel, the African meningitis belt. These have shown little difference in their major epidemiological characteristics from those of the epidemic described by Horn [2]. Nevertheless the similarity of these epidemics disguises the fact that over the years some subtle changes have been taking place.

The capsular serogroup of the meningococci responsible for the first African epidemics of cerebrospinal meningitis is not known. However, typing of isolates obtained from across Africa during the 1940s and 1950s yielded predominantly serogroup A isolates and at the time that Lapeyssonnie published his classical account of meningococcal disease in Africa in 1963 [3] it was generally assumed that all major epidemics of meningococcal disease in Africa were caused by meningococci belonging to this serogroup. Reports of epidemics in the 1970s in Nigeria [4] and Burkina Faso [5] in which a substantial number of isolates belonged to serogroup $\mathrm{C}$ showed that nonserogroup A meningococci have epidemic potential but these outbreaks were considered to be anomalies. This view was comprehensively demolished by the

\footnotetext{
* Author for correspondence: Professor B. Greenwood, Department of Infectious and Tropical Diseases, London School of Hygiene \& Tropical Medicine, Keppel St, London WC1E 7HT, UK.

(Email: brian.greenwood@1shtm.ac.uk)
}

occurrence in 2002 of a large epidemic of meningococcal disease in Burkina Faso which was caused predominantly by meningococci belonging to serogroup W135 [6]. This outbreak led to fears that a massive epidemic of serogroup W135 meningococcal meningitis might sweep across Africa with devastating effects in a non-immune population. Fortunately, this has not happened, but cases of W135 meningitis have continued to occur in Burkina Faso and neighbouring countries, often cohabiting with serogroup A meningococci. Recently, the story has taken an even more remarkable turn with the report of a major epidemic of over 4000 cases of meningococcal disease in Niger in 2006 caused in a large part by meningococci belonging to serogroup $\mathrm{X}$. In southwestern Niger meningococci belonging to this serogroup were responsible for $90 \%$ of cases [7].

The advent of major epidemics of serogroup W135 and serogroup X meningococcal infections in Africa raises a number of important questions. Where did the epidemic strains of bacteria come from?, what set off the epidemics caused by these strains?, and what can be done to contain them?

It might be anticipated that major epidemics of meningococcal disease in the African meningitis belt would be initiated by the introduction of a new strain from outside the belt, to which the local population had little immunity. There is evidence that this may have happened at least once in the case of serogroup A epidemics [8] and a possible explanation for the Burkina Faso epidemic is the introduction of the W135 strain by pilgrims returning from Mecca and Medina where serogroup W135 meningococci were responsible for a large proportion of meningococcal infections at the time of that outbreak [9]. However, acceptance of this apparently straightforward 
explanation is complicated by the fact that strains of serogroup W135 meningococci similar to the strain responsible for the epidemic in Burkina Faso have been circulating in West Africa for many years and were shown to be capable of causing invasive disease $[10,11]$. A similar situation pertains in relation to the serogroup X epidemic in Niger in 2006. Although this was the first large epidemic to be caused by meningococci of this serogroup, isolated cases and small outbreaks of serogroup $\mathrm{X}$ disease have been recorded in several West African countries including Niger and Ghana in the past $[12,13]$. What gave these bacteria, previously thought to be of relatively low virulence, the ability to cause major epidemics? Was this the acquisition of some new virulence factor by the epidemic strains of meningococci or could it have been due to some environmental change? No virulence factor has been identified to support the first hypothesis. Might the emergence of serogroup W135 and X meningococci be due to the selective advantage given to meningococci belonging to these serogroups by widespread vaccinations with serogroup $\mathrm{A}+\mathrm{C}$ meningococcal polysaccharides? In the past 20 years, $\mathrm{A}+\mathrm{C}$ meningococcal polysaccharide vaccines have been used extensively in the African meningitis belt to contain epidemics and many millions of doses have been given. Experience with pneumococcal conjugate vaccines has shown that vaccination can lead to serotype replacement so on first consideration this seems a credible explanation for the occurrence of W135 and X epidemics. However, there are several reasons which make this an unlikely explanation for what has occurred. First, there is little to suggest that in Africa meningococcal $\mathrm{A}+\mathrm{C}$ polysaccharide vaccines have any major impact on the prevalence of nasopharyngeal carriage of meningococci and it is in the nasopharynx that any selection would take place. Second, the use of serogroup $\mathrm{C}$ conjugate vaccines, which have had a major impact on carriage in the United Kingdom and elsewhere, has not so far been associated with any significant serotype replacement in disease or carriage, perhaps because of the generally low prevalence of carriage with serogroup $C$ strains in these communities. Finally, epidemiological studies in Burkina Faso and now in Niger have not shown any clear association at the regional level between attack rates with $\mathrm{W} 135$ or X meningitis and previous vaccination with $\mathrm{A}+\mathrm{C}$ polysaccharide vaccines. The role of prior vaccination cannot be excluded, as polysaccharide vaccines might have a more subtle effect on carriage than detected by simple cross-sectional surveys but, at present, this seems to be an unlikely cause of these epidemics.

Very few longitudinal studies of nasopharyneal carriage of meningococci have been undertaken in Africa but those that have, in particular an important study undertaken in northern Ghana [12], have shown that even over a fairly short period of time there are changes in the predominant serogroup of the meningococci present in the nasopharynx of asymptomatic carriers. These changes reflect natural cycles of infection and immunity that occur independently of any effect of vaccination. Perhaps epidemics of non-serogroup A meningococcal disease occur when the environmental factors that predispose to a major epidemic such as low absolute humidity, dust and possibly social factors occur at a time and place when a non-serogroup A meningococcus is at the peak of one of these natural waves.

The public health consequences of these recent changes in the epidemiology of meningococcal infection in Africa are serious. Although the sudden appearance of a serogroup W135 epidemic in Burkina Faso in 2002 was not followed by a massive epidemic across Africa, it is not clear why this did not happen as the limited number of studies that have been done have shown low background W135 bactericidal antibody concentrations in countries in the meningitis belt. An A,C,Y,W135 polysaccharide vaccine is available in limited amounts but it is too expensive for epidemic control as this vaccine is targeted primarily at protecting pilgrims and travellers to at-risk areas. This problem was overcome in an imaginative way by removing the serogroup $\mathrm{Y}$ polysaccharide component of the quadrivalent vaccine, thus invalidating it as a vaccine that could be used by pilgrims, but making it possible for the manufacturer to make this available for epidemic control at an affordable cost. This trivalent vaccine has been deployed widely in Burkina Faso with success. However, the advent of a serogroup $\mathrm{X}$ epidemic poses an even more difficult problem as there is currently no serogroup $\mathrm{X}$ vaccine commercially available even within a multivalent vaccine. Is the serogroup X strain responsible for the epidemic in Niger going to spread into neighbouring countries, for example northern Nigeria with its massive population or even to areas outside the meningitis belt? Should a crash programme be undertaken to produce a serogroup $\mathrm{X}$ vaccine and if so, who would be prepared to do this if the only market for the vaccine would be in Africa? 
On the other hand will serogroup X disease disappear with acquisition of naturally acquired immunity following widespread nasopharyngeal carriage with the epidemic strain? Unfortunately, our lack of knowledge of the factors that govern the peculiar epidemiology of meningococcal disease in Africa means that we can only guess.

\section{DECLARATION OF INTEREST}

None.

\section{REFERENCES}

1. Greenwood BM. Meningococcal meningitis in Africa. Transactions of the Royal Society of Tropical Medicine and Hygiene 1999; 93: 341-353.

2. Horn AE. Report on an investigation of cerebrospinal fever in the northern territories of the Gold Coast in 1908. Journal of Tropical Medicine and Hygiene 1908; 11: 358-365.

3. Lapeyssonie L. Cerebrospinal meningitis in Africa [in French]. Bulletin of the World Health Organization 1963; 28 (Suppl.): 3-114.

4. Whittle HC, et al. Group C meningococcal meningitis in the northern savannah of Africa. Lancet 1975; 1: 1377.
5. Broome CV, et al. Epidemic group C meningococcal meningitis in Upper Volta, 1979. Bulletin of the World Health Organization 1983; 61: 325-330.

6. Decosas J, Koama J-BT. Chronicle of an outbreak foretold: meningococcal meningitis W135 in Burkina Faso. Lancet Infectious Diseases 2002; 2: 663-765.

7. Boisier $\mathbf{P}$, et al. Meningococcal meningitis: unprecedented incidence of serogroup X-related cases in 2006 in Niger. Clinical Infectious Diseases 2007; 44: 657-673.

8. Achtman M. Molecular epidemiology of epidemic bacterial meningitis. Reviews of Medical Microbiology 1990; 1: 29-38.

9. Lingappa JR, et al. Serogroup W-135 meningococcal disease during the Hajj, 2000. Emerging Infectious Diseases 2003; 9: 665-671.

10. Denis F, et al. Emergence of meningococcal meningitis caused by W135 subgroups in Africa. Lancet 1982; ii: 1335-1336.

11. Kwara A, et al. Meningitis caused by a serogroup W135 clone of the ET-37 complex of Neisseria meningitidis in West Africa. Tropical Medicine and International Health 1998; 3: 742-746.

12. Gagneux SP, et al. Prospective study of a serogroup X Neisseria meningitidis outbreak in northern Ghana. Journal of Infectious Diseases 2002; 185: 618626.

13. Djibo S, et al. Outbreaks of serogroup X meningococcal meningitis in Niger 1995-2000. Tropical Medicine and International Health 2003; 8: 1118-1123. 